\title{
EFEITOS DE ORDEM, DO ENTREVISTADOR DO LOCAL E DO HORÁRIO DE COLETA DOS DADOS NOS RESULTADOS DE UMA PESQUISA PSICOSSOCIAL ${ }^{1}$
}

\author{
The effects determined by order, interviewer, place and time \\ during the data-collecting procedure on the results of a \\ social psychology research.
}

\author{
Marcos Emanoel Pereira ${ }^{2}$ \\ Ana Luiza Marques Fagundes ${ }^{3}$ \\ Joice Ferreira da Silva ${ }^{4}$ \\ Roberta Takei ${ }^{5}$
}

\section{Resumo}

Este estudo procura avaliar o efeito da ordem de apresentação dos estímulos, do entrevistador e do local de realização e horário de realização da coleta de dados nos resultados de uma pesquisa psicossocial. No primeiro caso, foi avaliado o efeito de ordem na apresentação dos estímulos. Os resultados evidenciaram a ausência de qualquer efeito de ordem, uma vez que independentes da ordem de apresentação das cenas, os resultados foram os mesmos. $\mathrm{O}$ entrevistador exerceu um efeito apenas modesto, pois das quatro entrevistadoras, observou-se diferença estatística significativa apenas nas respostas obtidas por uma delas. Em relação ao local das entrevistas, um único apresentou uma diferença significativa, e ainda assim, em apenas uma das cenas avaliadas. Por fim, no caso do horário de realização da coleta não foram observadas diferenças significativas nos resultados. Os dados indicam que os efeitos dos fatores contingenciais não parecem ser decisivos nos resultados da pesquisa.

Palavras-chave: Metodologia da Pesquisa; Pesquisa Psicossociológica; Survey; Experimentação.

1 Este trabalho recebeu suporte institucional do Programa de Iniciação Científica PIBIC CNPq./UFBa.

2 Professor Adjunto do Departamento de Psicologia/Programa de Pós-Graduação em Psicologia da Universidade Federal da Bahia.

3 Bolsista do Programa de Iniciação Científica PIBIC CNPq / UFBa, 2001/2002

Estudante do Curso de Psicologia da Universidade Federal da Bahia.

4 Bolsista do Programa de Iniciação Científica PIBIC CNPq / UFBa, 2002/2003

Estudante do Curso de Psicologia da Universidade Federal da Bahia

5 Estudante do Curso de Psicologia da Universidade Federal da Bahia

Endereço para contato: Rua Rodrigo Argollo, 293/502, Rio Vermelho, Salvador, BA. CEP: 41940-220

Email: emanoel@terra.com.br 


\section{Abstract}

This study is for evaluating the effects of the sequence in which the different stimulus are presented, the interviewer, the place of the experiment and the time of the data-collecting procedure. In the first instance, it was evaluated the effect determined by the order in a sequence of stimulus. The results revealed the absence of any effect determined by the sequence order, since they were the same independently of the variations applied to the sequence. The effect determined by the interviewer, by its turn, provoked only a single subtle result, because, considering the fact that there were four women acting as interviewer, only in the answers obtained by one of them it was noted significant statistics differences and, even so, only in one of the six scenes used in the experiment. Concerning the variation of places, from among the five sites where the data-collecting procedure was carried out, also only one presented a significant result difference. At last, as regards the hour when the data-collecting procedure is carried out, also only one of the four different hours presented a relevant statistic difference in result. Those results indicate, therefore, that the effect of the contingential factors does not seem to be decisive in spite of all the cautions adopted by the researcher.

Keywords: Methodology; Psychosocial Research; Survey Research; Experimental Research.

O psicólogo social dispõe de três grandes alternativas para a coleta de dados: a experimentação, a observação e o auto-relato (Coolican, 1999). Ninguém pode acusar de excesso de rigor a qualquer investigador pelo simples fato dele aderir ao pressuposto de que nenhuma dessas alternativas é perfeita e por admitir que todas apresentam vantagens e desvantagens. Esta postura, se facilita a vida do investigador, o obriga, no entanto, não apenas a conhecer um número razoável de métodos de investigação, como também a ser capaz de selecionar o mais apropriado para atender as circunstâncias particulares em que a investigação está sendo realizada. Desse modo, ele pode admitir que se a experimentação é um excelente meio para testar hipóteses alternativas sob condições controladas (Aronson, Wilson e Brewer, 1998), deixa muito a desejar quanto à possibilidade de ampliar as evidências obtidas a partir da pesquisa laboratorial para um contexto que não seja o do setting experimental. Da mesma forma, ele pode acreditar que se a observação facilita a coleta de dados em ambientes naturais, em contrapartida deixa a desejar no plano da qualidade dos dados, especialmente devido às dificuldades de codificação e categorização dos eventos observados (Foster, Laverty-Finch, Gizzo, Osantowski, 1999), requerendo ainda a participação de observadores treinados para uma avaliação independente e 0 domínio de procedimentos estatísticos de estimativa de confiabilidade que permitam determinar 0 índice de «concordância» entre os observadores. Por outro lado, ele também pode crer que se 0 auto-relato (Shadish, 2002) permite acessar um tipo de informação que dificilmente poderia ser obtido em um experimento ou por meio de técnicas observacionais, tal método se ressente muito da imprecisão dos relatos e convive com interpretações por demais subjetivas, sendo dependente, em última instância, de uma espécie de "química" entre pesquisador e pesquisado.

Por mais cuidadoso que seja o pesquisador com a escolha do método e com os procedimentos adotados durante a investigação, a pesquisa em psicologia social enfrenta um problema relativamente grave, que é a tendência a confiar em demasia nos resultados obtidos com amostras de estudantes universitários, na maior parte das vezes alunos de graduação que participam da pesquisa em troca de uma parcela dos créditos necessários para ser aprovado em uma disciplina, geralmente a cargo do próprio pesquisador (Sears, 1986). Em conseqüência desse problema, muitos psicólogos sociais reconhecem a necessidade de ampliar o plano amostral, incluindo participantes outros que não os estudantes de graduação. Afora isso, a seção de métodos dos periódicos tende a negligenciar as informações que permitam identificar o background demográfico dos participantes, bem como o sistema de incentivo à participação e 0 tempo de permanência do participante durante a atividade de coleta de dados (Korn e Bram, 1988).

O método por excelência voltado para a obtenção de dados generalizáveis para uma população mais ampla é o survey (Babbie, 1999). Este método exige a coleta de dados, geralmente obtidos através de questionários ou mesmo de entrevistas, de uma amostra de participantes, retirados de uma população claramente definida. Quando 
se pensa em pesquisa de survey, geralmente a idéia predominante é a de uma pesquisa bastante ampla, desenvolvida por um grande instituto e encomendada por uma organização com recursos suficientes para desembolsar somas altíssimas para investir na realização da pesquisa, cuja finalidade, quase sempre, se resume a atender aos interesses particulares dos patrocinadores. Alguns psicólogos sociais, entretanto, sugerem que não são necessários recursos financeiros extraordinários para a obtenção de dados de qualidade. Entre este ideal de obtenção de dados perfeitos e o mundo real existe, no entanto, uma grande distância, pois muitos erros podem impedir que uma amostra sirva como parâmetro para representar os atributos da população da qual ela é retirada. As diversas fontes de erro que podem impedir a obtenção de dados de qualidade estão organizadas em um modelo teórico denominado total survey error (Visser, Krosnick, e Lavrakas, 2000), de forma que 0 trabalho aqui apresentado procura ampliar o alcance desse modelo, indicando algumas fontes de erro que podem comprometer a qualidade dos dados obtidos em uma pesquisa psicossocial.

Um primeiro tipo de erro, o erro de cobertura, ocorre quando se observa um viés que faz com que uma parcela da população que se deseja investigar não seja incluída na amostra. Uma grande dificuldade relacionada com esse tipo de erro é a adoção de um delineamento de pesquisa impróprio para os objetivos da pesquisa. Assim, se o pesquisador deseja medir o impacto de um determinado evento que ocorreu entre a aplicação de dois surveys, ele deve adotar um design interseccional de medidas repetidas e não, por exemplo, um design de painéis, que possui a finalidade de avaliar o grau de estabilidade de um determinado constructo psicológico.

O segundo tipo, o erro de amostragem, refere-se às discrepâncias entre uma amostra de dados e os valores pretensamente verdadeiros que podem ser atribuídos à população de onde a amostra foi retirada e geralmente ocorre quando apenas um subconjunto da população definida é incluído no plano amostral. Nesse caso, é importante estabelecer a diferenciação entre uma amostra probabilística e uma não-probabilística (Sampieri, Collado e Lucio, 2000). Uma amostra é considerada probabilística quando os participantes são selecionados aleatoriamente e cada um tem uma chance conhecida e diferente de zero de ser seleciona- do. Um problema especialmente importante relacionado com as amostras probabilísticas é a quase impossibilidade de se conseguir, em algumas circunstâncias, uma lista completa capaz de incluir todos os elementos da população a ser estudada, o que torna praticamente impossível a obtenção de uma amostra por procedimentos aleatorizados. As alternativas para lidar com esse problema, tais como a seleção por área ou por meio de catálogo telefônico, introduzem várias outras fontes de erro, deparando-se sempre com o mesmo problema, dada a virtual impossibilidade de inclusão, com a mesma chance de serem escolhidos, de todos os elementos da população. As amostras não-probabilísticas são aquelas em que os participantes são selecionados de uma forma não-aleatória ou que alguns têm uma probabilidade desconhecida de serem selecionados. Uma forma bastante comum de obtenção de amostras não-probabilística é a conveniência, onde o pesquisador "seleciona" os participantes por um critério de facilidade, o que repercute de forma negativa na possibilidade de generalização e da formulação de inferências. Em outros casos, a seleção é voluntária, o que gera dificuldades na medida em que os participantes demonstram um maior interesse e muitas vezes já dispõem de algum conhecimento e, provavelmente, algum interesse sobre o assunto. $O$ método da bola de neve, em que um participante indica um outro, que por sua vez indica um próximo, até que a amostra seja finalizada, enfrenta uma dificuldade ainda mais grave, uma vez que viola 0 pressuposto de independência das observações. É importante assinalar, no entanto, que nem toda pesquisa científica possui a finalidade de obter dados que possam ser generalizados para a população como um todo ou mesmo para outro contexto que não aquele em que os dados foram coletados. Se, entretanto, esse é o objetivo, decerto a população a ser estudada tem de ser previamente identificada e caracterizada e a amostra a ser selecionada deve ser representativa da população como um todo. Geralmente este esforço é mais comum quando se trata de conduzir pesquisas cuja finalidade primordial é a descrição ou caracterização de um fenômeno ou evento. Se, ao contrário, o objetivo é o desenvolvimento de explicações sobre o fenômeno, a ênfase será bem maior na validade interna da pesquisa do que na determinação da probabilidade com o que o resultado possa vir a ser generalizado. 
O terceiro tipo, o erro por ausência de resposta, ocorre quando alguns membros da amostra não têm os seus dados coletados, como, por exemplo, nas circunstâncias em que o investigador não consegue localizar o participante. Uma contingência muito comum na qual esse erro se manifesta é aquela em que o participante, após ser abordado pelo pesquisador, se recusa a contribuir com a investigação. Outro tipo de dificuldade ocorre quando um participante começa a preencher o instrumento e, por alguma razão extraordinária - ou mesmo banal - deixa a tarefa incompleta. Em outras circunstâncias, especialmente quando se trata de instrumentos auto-aplicáveis enviados pelo correio ou deixados em mãos do participante para uma coleta posterior, uma grande dificuldade enfrentada pelo pesquisador é a de recuperar os questionários preenchidos.

Enfim, o quarto tipo, o erro de mensuração, ocorre nas circunstâncias em que se observa uma discrepância entre os atributos da população investigada e aquilo que foi efetivamente relatado pelos participantes (Schmidt e Hunter, 1996). A principal preocupação, nesse caso, embora não única, é com os diversos tipos de erro aleatórios e sistemáticos que podem comprometer o registro de um evento psicológico. Muitas dificuldades começam com a simples decisão de se adotar um instrumento aberto ou fechado. No caso de instrumentos fechados, o investigador deve decidir se as alternativas de respostas serão obtidas através de ranking ou de rating. No caso das escalas de atitudes, ele deverá decidir claramente quantos itens de respostas serão incluídos e se entre as alternativas será apresentado um ponto médio ou neutro. Outras questões significativas que podem gerar erros estão relacionadas com a terminologia usada para apresentar a assertiva a ser avaliada, assim como com a ordem de apresentação das questões, bem como a ordem de apresentação das alternativas de resposta (Myers, 1999). Além desses fatores, também podem contribuir para a obtenção de dados de baixa qualidade a ausência de pré-teste do instrumento, assim como procedimentos inadequados de coleta de dados. Outra fonte de erro de mensuração relaciona-se com o próprio participante, dado que qualquer resposta apresentada exige o processamento de um número razoável de informações, uma vez que o participante só será incluído caso consiga entender as instruções e o enunciado, retirar da memória o significado geral do que foi exposto, definir uma estratégia de resposta, selecionar entre os scripts disponíveis 0 mais adequado para a situação e formular a resposta, transformando-a em um comportamento aberto. Ora, várias fontes de erro podem se manifestar em todo esse processo. Além disso, nenhum pesquisador deve deixar de considerar a tendência do participante em «editar» a resposta, seja devido à desejabilidade social, às estratégias de autoapresentação ou devido às pressões situacionais as quais se encontra sujeito. Uma outra fonte de comprometimento da qualidade dos dados relaciona-se com o pesquisador. Nesse caso, há de se considerar uma série de restrições, especialmente aquelas que envolvem o background étnico e de gênero, dado que muitos participantes não se sentem à vontade quando são entrevistados ou mesmo quando são obrigados a interagir com os membros de uma determinada categonia social. Afora isso, mesmo que esses efeitos sejam controlados, os pesquisadores devem ser treinados ou bem instruídos em relação às rotinas a serem adotadas durante o procedimento de coleta de dados. Enfim, o próprio processo de coleta dos dados pode gerar novos erros, especialmente se o investigador não se assegurar de manter constantes as condições em que a coleta é realizada.

\section{Objetivo}

As análises aqui apresentadas foram realizadas a partir dos dados obtidos no contexto de uma investigação sobre os estereótipos, mais especificamente, na avaliação das estratégias usadas por adultos para a codificação lingüística de cenas visualmente apresentadas. Nesse caso, os participantes avaliaram seis cenas, três positivas e três negativas.

Se o objetivo da pesquisa acima referida foi a de testar as estratégias lingüísticas usadas para codificar cenas visualmente apresentadas em que aparecem membros do seu próprio grupo e de outras categorias sociais, o objetivo fundamental do presente trabalho é investigar o efeito de quatro fatores, incluídos no quarto tipo de erro característico do modelo do total survey error, na qualidade dos dados obtidos. O primeiro fator que consideramos foi a influência do experimentador. Nesse caso, o objetivo central foi o de determinar a existência de diferenças significativas nos resul- 
tados obtidos pelas quatro experimentadoras que participaram da coleta de dados. Posteriormente, buscamos determinar se o local e o horário da coleta dos dados influenciaram os resultados, mediante a comparação dos resultados obtidos na coleta de dados em diferentes locais e horários. Nesse caso, a nossa finalidade foi a de determinar se os padrões de resposta sofreram alguma influência do local e do horário em que a coleta de dados foi realizada. $\mathrm{O}$ quarto fator considerado foi a influência da ordem de apresentação dos itens. Nesse caso, os seis estímulos avaliados foram apresentados em uma ordem diferenciada e procuramos determinar se essa diferença na posição produziu alguma mudança na avaliação realizada pelos participantes da pesquisa.

Fundamentalmente, as nossas hipóteses básicas de trabalho sustentam que, i) por se tratar de uma formulação baseada em uma teoria que pretende ser universal, portanto aplicável aos membros das diversas categorias sociais, independentes da condição geográfica, histórica, cultural e social; ii) pelo fato dos dados terem sido apresentados de uma forma sistemática; e iii) pelos entrevistadores se portarem de acordo com um plano de atuação previamente definido, dificilmente poder-se-ia esperar qualquer diferença significativa nos resultados obtidos pelas quatro entrevistadoras (hipótese 1), nos diferentes locais e horários da coleta de dados (hipóteses 2 e 3), assim como no efeito de ordem de apresentação das cenas (hipóteses 4).

\section{Métodos}

Nesta seção serão apresentadas as informações relativas à constituição da amostra, aos procedimentos e aos instrumentos utilizados durante a investigação.

\section{Amostra}

A amostra foi constituída por 83 participantes. A distribuição por gênero indicou a participação de 49 (59,0\%) indivíduos do sexo masculino, $33(39,8 \%)$ do sexo feminino, não sendo possível registrar, durante o procedimento de coleta de dados, o gênero de um participante da pesquisa. A distribuição por faixa etária pode ser observada na tabela 1 :
Tabela 1: distribuição dos participantes por faix a etária

\begin{tabular}{|llr|}
\hline Faixa etária & N & \% \\
\hline 12 a 16 anos & 03 & 3,6 \\
17 a 21 anos & 22 & 26,5 \\
22 a 26 anos & 19 & 22,9 \\
27 a 31 anos & 13 & 15,7 \\
32 a 36 anos & 06 & 7,2 \\
37 a 41 anos & 05 & 6,0 \\
42 a 46 anos & 05 & 6,0 \\
47 a 51 anos & 03 & 3,6 \\
52 a 56 anos & 03 & 3,6 \\
Acima de 56 anos & 04 & 4,8 \\
\hline Total & $\mathbf{8 3}$ & $\mathbf{1 0 0 , 0}$ \\
\hline
\end{tabular}

Em relação ao grau de escolaridade dos participantes, a distribuição, conforme se observa na tabela 2 , teve uma amplitude bastante alta, atingindo a todas as faixas de escolaridade, embora tenha sido observado um predomínio entre os participantes com o segundo grau completo e 0 terceiro incompleto.

\section{Tabela 2: distribuição dos participantes por grau de escolaridade}

\begin{tabular}{|lrr|}
\hline Grau de escolaridade & N & \% \\
\hline Ensino Básico incompleto & 12 & 14,5 \\
Ensino Básico completo & 06 & 7,2 \\
Ensino Médio incompleto & 14 & 16,9 \\
Ensino Médio completo & 25 & 30,1 \\
Ensino Superior incompleto & 17 & 20,5 \\
Ensino Superior completo & 09 & 10,8 \\
\hline
\end{tabular}

Em suma, pode-se notar uma distribuição bastante abrangente em relação ao gênero, à faixa etária e ao grau de escolaridade dos participantes. 


\section{Procedimentos}

A metodologia adotada neste trabalho foi a experimental. $\mathrm{O}$ experimento utilizou um delineamento fatorial $2 \times 2 \times 2$ (etnia do participante, branca ou negra; grupo étnico do personagem da cena, ingroup ou outgroup; e tipo de cena, positiva ou negativa), sendo o último fator de medida repetida. Os dados foram obtidos através de uma escala em que se apresentou uma série de alternativas e o participante indicou a que melhor retratava a natureza da cena visualmente apresentada.

O experimento utilizou um caderno de apresentação em que estavam desenhadas seis cenas, três socialmente positivas (devolver a carteira perdida de um transeunte; participar de uma roda de capoeira; ajudar uma senhora idosa a atravessar a rua) e três negativas (dirigir-se a um grupo sentado em uma mesa de bar; ficar à espreita em uma praia, esperando uma oportunidade para cometer um furto; atingir com um tapa uma outra criança de menor estatura e idade). Foram elaborados dois conjuntos de cenas em que todos os personagens centrais eram crianças, sendo que cada conjunto retratava os personagens centrais como de etnia branca ou negra. Para evitar o efeito de ordem, houve um balanceamento na apresentação, de forma que a primeira cena apresentada a um participante foi a última apresentada ao seguinte. Com base no acima exposto, foram constituídos quatro grupos experimentais, assim distribuídos: 1) participantes de etnia branca, avaliando personagens de etnia branca; 2) participantes de etnia branca, avaliando personagens de etnia negra; 3) participantes de etnia negra, avaliando personagens de etnia branca; e 4) participantes de etnia negra, avaliando personagens de etnia negra.

O critério para a seleção dos participantes foi a conveniência. Todos eles foram aborda- dos, preferencialmente quando se encontravam sozinhos, em locais públicos e convidados a participar do experimento.

\section{Instrumentos}

As cenas foram especialmente elaboradas para esse estudo e uma delas encontra-se reproduzida nos itens a1 e a2 do anexo 1. As alternativas de resposta incluíam quatro itens e exprimiam os diferentes níveis de abstração com que as cenas podiam ser lingüisticamente representadas, conforme se observa nos item b1 do anexo 1 .

\section{Análise e discussão dos resultados}

Os resultados foram computados usando como critério a média das respostas em relação ao grau de abstração na avaliação das cenas. Para fins de análise, embora o delineamento sugerisse a necessidade de considerar dois grupos de cenas, as positivas e as negativas, a estimativa de confiabilidade mensurada através do alpha de Cronbach (0,51 e 0,19 respectivamente) foi bastante aquém dos valores desejáveis, de forma que consideraremos os resultados de cada uma das cenas em separado.

\section{0 efeito do ex perimenta dor}

Quatro experimentadoras conduziram toda a coleta de dados, trabalhando de forma separada, no mesmo local e nos mesmos horários. Os resultados das avaliações das cenas obtidos por cada uma delas estão representados na tabela 3 . 
Tabela 3: Média das respostas obtidas pelos entrevistadores em cada uma das cenas

\begin{tabular}{|c|c|c|c|c|c|}
\hline $\begin{array}{l}\text { Entrevistador } \\
\text { Adjetivo }\end{array}$ & $\begin{array}{l}\text { Entrevistador } \\
\text { A } \\
(\mathrm{n}=15)\end{array}$ & $\begin{array}{l}\text { Entrevistador } \\
\text { B } \\
(n=20)\end{array}$ & $\begin{array}{l}\text { Entrevistador } \\
\text { C } \\
(n=24)\end{array}$ & $\begin{array}{l}\text { Entrevistador } \\
\text { D } \\
(\mathrm{n}=24)\end{array}$ & $\mathbf{P}$ \\
\hline Honesto & 1,67 & 2,20 & 1,92 & 2,08 & .293 \\
\hline Capoeirista & 2,80 & 2,55 & 2,75 & 2,42 & .443 \\
\hline Prestativo & 2,47 & 2,65 & 2,88 & 2,58 & .737 \\
\hline Pedinte & 3,87 & 3,30 & 3,71 & 2,92 & .003 \\
\hline Ladrão & 3,53 & 3,55 & 3,71 & 3,75 & .664 \\
\hline Agressivo & 2,26 & 2,70 & 2,17 & 2,04 & .272 \\
\hline
\end{tabular}

A análise estatística através da ANOVA indica que em apenas uma das cenas foi observada uma diferença significativa $(p=.003)$ na coleta de dados realizada pelas quatro experimentadoras, no caso, a cena em que o personagem central foi representado como pedinte. O teste post hoc Tukey HSD indicou que os resultados obtidos pela experimentadora D apresentaram uma diferença significativa quando comparados com os obtidos pelas experimentadoras A $(p=.007)$ e C $(p=.011)$. Um elemento importante capaz de explicar essa diferença nos resultados obtidos relaciona-se com a própria apresentação da cena. A análise dos dados do questionário abertos (não considerados nesse artigo) indica uma grande ambigüidade em relação à interpretação da cena, dado que se muitos participantes sugeriram explicitamente que se tratava do ato de pedir, outros interpretaram a cena como uma ação em que um garoto apontava para a mesa, sem se referir claramente ao que poderia ser interpretado como mendicância. Alguns participantes, inclusive, chegaram ao extremo de assinalar que o personagem da cena estava tentando convencer aos amigos que se encontravam na mesa a parar de beber.

Em linhas gerais, podemos sugerir que os resultados indicam que o efeito do entrevistador foi bastante reduzido, manifestando-se apenas em uma das seis cenas avaliadas e, mesmo assim, nos resultados coletados por uma das entrevistadoras.

\section{0 efeito do local}

A coleta de dados foi realizada em quatro locais diferentes. O primeiro deles, o Largo de Santana, no bairro do Rio Vermelho, é um local de grande concentração de turistas e de profissionais liberais, trabalhadores e estudantes que aproveitam o fim de tarde em um conjunto de bares que organizados a partir da clientela de uma famosa baiana de acarajé. A Praça da Piedade, o segundo local onde os dados foram coletados, situa-se na região central da cidade, sendo especialmente ocupada, no horário em que a coleta de dados foi realizada, por aposentados, por populares que se dirigem ao trabalho, geralmente nas lojas da Avenida Sete e adjacências, e por pessoas que se preocupam apenas em flanar pelo centro da cidade. O terceiro local, a Estação Rodoviária da cidade do Salvador, é um local de grande movimento, freqüentado especialmente pela população que se desloca da capital para as cidades do interior e de outros estados. Enfim, o último local de coleta de dados, o Shopping Center Iguatemi, é provavelmente o principal centro de consumo da capital baiana, freqüentado prioritariamente por pessoas da classe média. Os resultados obtidos na avaliação das cenas coletadas nos diversos locais em que a pesquisa foi realizada indica, conforme pode ser observado na tabela 4, que em apenas uma das cenas foi obtida uma diferença significativa, enquanto outra obteve uma diferença estatística apenas marginal. 


\section{Tabela 4: Média das respostas obtidas em diferentes locais para cada uma das cenas}

\begin{tabular}{|llllll|}
\hline Local & $\begin{array}{l}\text { Rio } \\
\text { Vermelho } \\
\text { Adjetivo }\end{array}$ & $\begin{array}{l}\text { Praça da } \\
\text { Piedade } \\
\mathbf{( n = 1 9 )}\end{array}$ & $\begin{array}{l}\text { Estação } \\
\text { Rodoviária } \\
(\mathbf{n = 3 6 )}\end{array}$ & $\begin{array}{l}\text { Shopping } \\
\text { Iguatemi } \\
\text { (n=19) }\end{array}$ & P \\
\hline Honesto & 2,11 & 1,90 & 1,89 & 2,21 & .550 \\
Capoeirista & 2,67 & 2,79 & 2,36 & 2,90 & .107 \\
Prestativo & 3,44 & 2,74 & 3,67 & 3,79 & .084 \\
Pedinte & 3,79 & 3,68 & 3,08 & 3,58 & .038 \\
Ladrão & 3,89 & 3,37 & 3,67 & 3,78 & .148 \\
Agressivo & 2,67 & 2,00 & 2,36 & 2,21 & .503 \\
\hline
\end{tabular}

A análise, através do Tukey HSD, da cena em que o personagem era apresentado como pedinte, indicou uma diferença estatística apenas marginal $(\mathrm{p}=.091)$ nos resultados obtidos em dois locais distintos, a Rio Vermelho e a Estação Rodoviária. Este resultado talvez possa indicar uma diferença no perfil demográfico dos participantes, uma vez que os participantes abordados no Rio Vermelho apresentavam uma condição social bem diferente dos abordados na Estação Rodoviária, já que possuíam um nível educacional e econômico aparentemente mais alto. Um outro fator a se considerar, diretamente relacionado com a natureza da cena em que obteve a diferença estatística, é a grande quantidade de pedintes e vendedores ambulantes, especialmente crianças, que se aglomeram no Rio Vermelho. Estudos sobre o processo de estereotipização indicam que o maior contato entre o percebedor e membros do grupo alvo leva a uma maior familiaridade com os membros do grupo alvo dos estereótipos, o que pode, por sua vez, levar a uma maior individualização durante 0 processo de julgamento social, o que, em certa medida, pode ter ocorrido quando os participantes do Rio Vermelho avaliaram a cena em que o personagem era representado como pedinte. De qualquer modo, parece evidente que o efeito do local da coleta de dados exerceu um efeito bastante modesto nos resultados.

\section{Efeito do horário}

Os dados foram coletados em cinco horários diferentes, $9 \mathrm{~h} 30$ e 10 horas da manhã, 14 e 15 horas e 18 horas. Para fins de análise foram considerados dois grupos, o da coleta de dados no horário matutino e o segundo que agrupou os dados obtidos nos períodos vespertino e noturno. A análise em separado de cada uma das cenas indicou uma diferença estatística significativa em duas delas, uma na qual o personagem é retratado como pedinte e em uma outra, na qual o personagem central é representado como ladrão. Mesmo assim, adotamos a estratégia de agrupar os dados, uma vez que o teste post hoc Tukey HSD indicou que as diferenças estatísticas entre as duas cenas foram apenas marginais (.066 e .079) e exclusivamente nos horários de $9 \mathrm{~h} 30$ e 10 horas. 
Tabela 5: Média das respostas obtidas para cada uma das cenas em diferentes horários

\begin{tabular}{|llll|}
\hline Horário & $\begin{array}{l}\text { matutino } \\
\mathbf{( n = 3 5 )}\end{array}$ & $\begin{array}{l}\text { Vesp./noturno } \\
\mathbf{( n = 4 3 )}\end{array}$ & $\mathbf{P}$ \\
Adjetivo & & 687 \\
\hline Honesto & 2,00 & 1,89 & .899 \\
Capoeirista & 2,44 & 2,79 & .824 \\
Prestativo & 2,50 & 2,74 & .426 \\
Pedinte & 2,88 & 3,68 & .800 \\
Ladrão & 3,94 & 3,37 & .138 \\
Agressivo & 2,13 & 2,00 & \\
\hline
\end{tabular}

Estes resultados indicam, portanto, a ausência de qualquer influência do horário nas respostas apresentadas pelos participantes do estudo.

\section{Efeito de ordem de apresentação das cenas}

Para determinar a influência da ordem de apresentação das cenas, houve um rodízio no arranjo da apresentação dos estímulos, de forma que o estímulo apresentado em primeiro lugar em uma coleta, tornava-se o último a ser apresentado na coleta seguinte. A tabela 6 sistematiza a média dos resultados obtidos, considerando os resultados quando cada cena foi apresentada em primeiro lugar.
Considerando que um dos fatores que mais influenciam o efeito de ordem é o acréscimo da compreensão da tarefa que ocorre na medida em que o participante passa a responder mais questões (Knowles, 1988), pode-se argumentar que tal efeito não se manifestou no presente caso, dada a ausência de qualquer diferença significativa entre as cenas.

\section{Conclusões}

Entre o mundo do laboratório e o mundo real existe uma distância quase intransponível para os psicólogos sociais. $\mathrm{O}$ rigor do laboratório, embora permita o teste das hipóteses de forma mais cuidadosa, em contrapartida torna-se estéril quan-

\section{Tabela 6. Média das respostas obtidas de acordo com a ordem de apresenta- ção da cena}

\begin{tabular}{|llllllll|}
\hline Ordem & $\begin{array}{l}\text { Honesto } \\
\text { (n=15) }\end{array}$ & $\begin{array}{l}\text { Capoeirista } \\
\text { (n=15) }\end{array}$ & $\begin{array}{l}\text { Prestativo } \\
(\mathbf{n = 1 3 )}\end{array}$ & $\begin{array}{l}\text { Pedinte } \\
(\mathbf{n = 1 5 )}\end{array}$ & $\begin{array}{l}\text { Ladrão } \\
(\mathbf{n = 1 2 )}\end{array}$ & $\begin{array}{l}\text { Agressivo } \\
(\mathbf{n = 1 3 )}\end{array}$ & $\mathbf{p}$ \\
\hline Honesto & 2,07 & 2,00 & 1,92 & 2,27 & 1,58 & 2,00 & .501 \\
Capoeirista & 2,60 & 2,33 & 2,85 & 2,47 & 2,83 & 2,69 & .569 \\
Prestativo & 2,80 & 3,00 & 3,00 & 2,60 & 2,17 & 2,31 & .329 \\
Pedinte & 3,20 & 3,33 & 3,92 & 3,27 & 3,42 & 3,38 & .392 \\
Ladrão & 3,60 & 3,73 & 3,92 & 3,53 & 3,83 & 3,85 & .447 \\
Agressivo & 2,27 & 2,33 & 2,15 & 2,87 & 1,75 & 2,15 & .237 \\
\hline
\end{tabular}


do refletimos sobre as possibilidades de generalização das evidências aí obtidas para o mundo real. Muitos psicólogos sociais sugerem que essa necessidade de se encontrar alternativas que possam complementar - e esclarecer - muitas das questões deixadas em aberto pela pesquisa experimental de laboratório deve ser atendida apenas na medida em que seja encontrada alguma probabilidade de controlar de forma rigorosa a qualidade dos dados obtidos fora do ambiente do laboratório. Como esses problemas podem ser enfrentados? De acordo com Schartz (1999), diversas técnicas foram desenvolvidas e se utilizadas de forma adequada durante o procedimento de pré-testagem do instrumento, muitos problemas podem ser evitados.

Essa preocupação com a qualidade dos dados, assim como com a generalização dos resultados, situa em um plano de igualdade a validade interna e a validade ecológica. $\mathrm{O}$ presente estudo apresenta algumas evidências de que muitos dados obtidos em laboratório podem ser alcançados em ambientes naturais sem que a qualidade venha a ser comprometida. $\mathrm{O}$ efeito do entrevistador, do local e do horário, assim como da ordem de apresentação dos estímulos mostraram exercer um efeito modesto nos resultados. Claro que vários outros fatores devem ser estudados para que se chegue a uma conclusão mais confiável sobre o assunto, mas de qualquer modo, os resultados aqui apresentados podem servir como indicadores de que o psicólogo social pode ter alguma tranqüilidade no momento em que deixa o ambiente seguro e frio do laboratório e passa a ocupar outros espaços de investigação.

\section{Referências}

ARONSON, E., Wilson, T. \& Brewer, M. (1998). Experimentation in social psychology. In D.

GILBERT, S. Fiske e G. Lindzey (1998). The handbook of social psychology, fourth edition, Boston: Mc-Graw-Hill.

BABBIE, E. (1999). Métodos de pesquisa de survey. Belo Horizonte: Editora UFMG
COOLICAN, H. (1999). Introduction to research methods and statistics in psychology. London: Hodder \& Stoughton.

FOSTER, S., Laverty-Finch, C., Gizzo, D. \& OsantoWSKI, J. (1999) Practical Issues in Self-Observation. Psychological Assessment. 11, (4), 426-438.

KORN, J e Bram, D. (1988). What Is Missing in the Method Section of APA Journal Articles? American Psychologist, 3, (12), 1091-1092.

KNOWLES, E. (1988). Item context effects on personality scales: measuring changes the measure. Journal of Personality and Social Psychology, 70, 1080-1090.

MYERS, D. (1999). Social Psychology. Boston: McGraw-Hill

SAMPIERI, R., Collado, C. \& Lucio, P. (2000). Metodología de la investigación. Mexico: McGrawHill.

SCHWARZ, N. (1999). Self-reports: how the questions shape the answers. American Psychologist, 54, (2), 93-105.

SEARS, D (1986). College sophomores in the laboratory: Influences of a narrow data base on psychology's view of human nature. Journal of Personality and Social Psychology, 51, 515-530.

SCHMIDT, F. \& Hunter, J. (1996) Measurement Error in Psychological Research: Lessons From 26 Research Scenarios. Psychological Methods, 1, (2), 199-223.

SHADISH, W. (2002). Revisiting Field Experimentation: Field Notes for the Future. Psychological Methods, 7, (1), 3-18.

VISSER, P., Krosnick, J. \& Lavrakas, P. (2000). Survey Research. In H. Reis e C. Judd. Handbook of research methods in social and personality psychology. Cambridge: Cambridge University Press 